\title{
TECNOLOGÍA DE ATMÓSFERAS CONTROLADAS PARA FRUTAS Y HORTALIZAS, CASO DE ESTUDIO: MODELO AGUACATE (Persea Americana) VARIEDAD HASS
}

\author{
Diego Montoya \\ Escuela de Postgrados, Universidad Nacional Abierta y a Distancia, Calle 53 No. 14 - \\ 39. Bogotá, Colombia. Teléfono: 3460088 Fax: 3472556.
}

\begin{abstract}
Resumen
La tecnología de atmósferas controladas (AC) se utiliza en cuartos fríos para el almacenamiento, o en contenedores para el transporte, de productos hortofrutícolas. Esta técnica estabiliza la atmósfera que rodea el producto en porcentajes precisos con relación al $\mathrm{O}_{2}$ y al $\mathrm{CO}_{2}$ presentes en ella, dependiendo de la variedad vegetal a controlar. Para el aguacate (Persea Americana) como fruta climatérica y concretamente para la variedad Hass, producto de exportación mundial, la conservación en Atmósfera Controlada es innovadora para el mantenimiento de su calidad óptima.
\end{abstract}

Palabras Claves: Tecnología, Atmósfera, Controlada, Frutas, Aguacate.

\begin{abstract}
The controlled atmosphere technology $(A C)$ is used in cold quarters for the storage, or in containers for the transport, of vegetables and fruits products. This technique stabilizes the atmosphere that surrounds the product in precise percentage with relation to the present $\mathrm{O}_{2}$ and $\mathrm{CO}_{2}$ in her, depending on the vegetal variety to control. For the avocado (American Persea) like climacterical fruit and concretely for the Hass variety, product of world-wide export, the conservation in AC is innovating for the maintenance of its optimal quality.
\end{abstract}

Key Words: Technology, Atmosphere, Control, Fruit, Avocado.

\section{Introducción}

Las pérdidas poscosecha de frutas y hortalizas a nivel mundial alcanzan del 30 al $40 \%$, siendo mayores en los países en vía de desarrollo (FAO 2002), por lo que se hace necesario mejorar cada vez más las técnicas de almacenamiento y conservación de estos productos.

Las diferentes variedades hortofrutícolas se conservan en un ambiente adecuado con el objetivo de reducir las pérdidas por su deterioro durante el proceso de mercadeo. Cada producto tiene condiciones ideales de almacenamiento (temperatura, humedad relativa, aire que lo rodea) de acuerdo a sus características particulares (respiración, transpiración, producción de etileno).

Al bajar la temperatura de los productos se hace más lento el proceso metabólico, pero si este se acompaña de la modificación de la atmósfera que lo rodea, se puede retardar mucho más dicho proceso.

\section{Desarrollo histórico de las atmósferas controladas}

Los primeros intentos de trabajo con $\mathrm{AC}$ en vegetales fueron hechos por West y Kidd en 1918 en la Universidad de Cambridge, donde se estudió la posibilidad de utilizar AC como alternativa diferente al de ser un complemento para la refrigeración. En 1926 
Kidd y West hallaron los beneficios que se obtenían al controlar el $\mathrm{O}_{2}$ y el $\mathrm{CO}_{2}$ y la temperatura sobre algunas variedades vegetales. Ziegler y Nichds en 1942 descubrieron la técnica de regulación sobre los gases componentes de la AC; pero solamente hasta 1980 se hace automáticamente el control de gases. En 1976 North y Cocbum estudiando el almacenamiento de manzanas cox encontraron concentraciones del $1.25 \%$ para el $\mathrm{O}_{2}$ y del $1.00 \%$ para el $\mathrm{CO}_{2}$ que hacían óptima su conservación (Revista Calor y frío 2002).

Actualmente la utilización de sistemas automatizados para el control de AC hace que su manejo sea conceptualmente muy simple ofreciendo ventajas sobre la regulación manual como: precisión, continuidad en el proceso de almacenamiento y ahorro en la mano de obra.

\section{Parámetros de diseño de una Cámara de Atmósfera Controlada (CAC)}

En el diseño de CAC se deben tener en cuenta varios factores:

- Uso. Una CAC puede servir como almacén por semanas o meses, como bodega temporal o como centro de distribución diaria.

- Aspectos financieros. Se deben calcular los costos de su construcción y mantenimiento y estimar los futuros beneficios económicos que se obtendrán.

- Localización. En este parámetro se debe tener en cuenta: la cercanía de los lugares de producción, la ubicación de los potenciales mercados, y facilidad de medios de transporte.

- Tamaño de la CAC. Las capacidades de enfriamiento, de modificación de la atmósfera y del almacenamiento requerido, son características separadas, pero las tres determinan el tamaño de la cámara.

- Aspectos relacionados con la obra física. Aquí se incluyen los aspectos técnicos que garanticen su hermeticidad a saber: materiales y aislamiento, donde el material más utilizado es el poliuretano; las juntas entre el piso y las paredes no deben ser separadas para evitar pérdida de sellado; las puertas deben se de cerrado hermético; se deben colocar topes contra la pared y el piso de tal manera que garanticen la circulación de aire interno.

- Cálculo de la carga de calor. La temperatura óptima de almacenamiento debe ser mantenida continuamente para garantizar la conservación de los productos el tiempo que se espera, lo cual requiere calcular la correspondiente capacidad de refrigeración a las condiciones de carga más exigentes a las cuales trabajará la CAC.

- Cálculo de la mezcla de gases. Para generar la AC se tienen en cuenta las características de cada producto. En general de acuerdo a estudios experimentales se recomiendan los siguientes porcentajes de gases para frutas (Guillen, 1998): 3 - \%\% de $\mathrm{O}_{2}, 3-5 \%$ de $\mathrm{CO}_{2}, 85-95 \%$ de $\mathrm{N}_{2}$.

\section{Funcionamiento de una cámara de atmósfera controlada}

$\mathrm{Al}$ colocar en funcionamiento una CAC se precisa tener tres consideraciones: empleo de bajas temperaturas (sin llegar a la congelación, ni producir daños por frío); mantener elevada humedad relativa, evitando la condensación del agua sobre los productos, y observar la renovación apropiada y controlada del aire ambiente. Tener presente estos 
aspectos hace que en el producto vegetal se frene su metabolismo, se prolongue su vida útil y se preserve su calidad para el consumo (Artes, 1999).

En general el funcionamiento de una CAC consiste en mantener bajo observación permanente, porcentajes determinados de $\mathrm{O}_{2}, \mathrm{CO}_{2}$; y la temperatura y humedad relativa necesarias en una bodega hermética refrigerada, la cual debe contar con los equipos de medición y control para el monitoreo de los anteriores parámetros.

Existen varios sistemas de AC entre los cuales se pueden citar:

- $\quad$ TECTRON, el cual se basa para su funcionamiento en un quemador catalítico de una mezcla de aire y propano, lo cual genera una atmósfera rica en $\mathrm{N}_{2}$ pero pobre en $\mathrm{O}_{2}$, la cual debe estar contenida en un espacio sellado. El contenido de $\mathrm{CO}_{2}$ se regula con un absorbedor de carbón activado.

- HOLEC, este sistema se basa en la producción de $\mathrm{N}_{2}$ a través del craking del amoniaco como medio para disminuir el $\mathrm{O}_{2}$. La utilización de $\mathrm{N}_{2}$ ofrece más seguridad en la operación de las CAC, así como una mayor rapidez en la disminución del contenido de $\mathrm{O}_{2}$ en las cámaras.

- $\quad$ AC convencional, se basa en la introducción de aire desde el exterior de la cámara para mantener el nivel de $\mathrm{O}_{2}$. El nivel de $\mathrm{CO}_{2}$ se mantiene removiéndolo desde la cámara hacia el exterior, mediante absorbedores. Concentraciones de $\mathrm{CO}_{2}$ mayores a las permitidas por una variedad determinada pueden generar oscurecimiento en la pulpa y ruptura de los tejidos.

- AC rápida, se basa en el control de las concentraciones de los gases inmediatamente después de sellar herméticamente la cámara. Los niveles de gases se pueden mantener mediante los procedimientos utilizados en la AC convencionales o sistematizando el proceso.

- $\quad \mathrm{AC}$ de ultra bajo $\mathrm{O}_{2}$, es una cámara de $\mathrm{AC}$ rápida de niveles de $\mathrm{O}_{2}$ menores del $1 \%$, donde no se usan altos niveles de $\mathrm{CO}_{2}$. En esta cámaras se necesita un control minucioso para evitar que e nivel de $\mathrm{O}_{2}$ disminuya y cause daños en el producto. Bajos niveles de $\mathrm{O}_{2}$ ocasionan la aparición de sabores extraños debido a la oxidación anaeróbica de los carbohidratos. Las reducciones de $\mathrm{O}_{2}$ se pueden hacer por llama abierta, quemadores catalíticos, quemadores de amoníaco, $\mathrm{N}_{2}$ líquido o gaseoso, utilización de membranas huecas para separar $\mathrm{O}_{2}$ y $\mathrm{N}_{2}$.

- $\quad \mathrm{AC}$ de bajo etileno. Se basa en el funcionamiento de una AC rápida que mantiene controles precios de etileno los cuales no pueden ser mayores de $1 \mathrm{ppm}$.

\section{Ventajas y consideraciones a tener en cuenta en la utilización de CAC}

Las ventajas que se pueden obtener combinando la $\mathrm{AC}$ en una cámara refrigerada son las siguientes:

- Alargamiento de la vida de conservación.

- Creación de nuevos segmentos de mercado para las frutas exóticas.

- Los parásitos que se encuentran en la carga mueren o su desarrollo resulta muy retardado (reducción del tratamiento después de la cosecha, calidad, costos).

- Continuamente se obtienen datos de control de la temperatura, del $\mathrm{O}_{2}$, del $\mathrm{CO}_{2}$ y de la humedad relativa (calidad). 
- Las frutas pueden transportarse en un grado de madurez más avanzado que anteriormente, de modo que tiene mejor sabor (calidad).

- La duración del transporte de las frutas se puede alargar considerablemente, es decir que las frutas hasta ahora transportadas, sobre todo por vía aérea, pueden transportarse gracias a ésta tecnología por vía terrestre o marítima.

- Reducción de las pérdidas durante el transporte (costos).

Para responder por el mantenimiento de productos vegetales bajo AC se deben tener en cuenta los siguientes criterios de calidad:

- El producto vegetal debe estar sano y respetar todas las normas de higiene.

- Son esenciales las prácticas rigurosas y sistemáticas del control de higiene, desde la recepción de los productos y durante el almacenaje, distribución y venta.

- Condiciones higiénico-sanitarias de manipuladores, ambientes, equipos, como de la red de distribución y de los gases.

- Disponer de un equipo de acondicionamiento apropiado, con respecto al funcionamiento y control de la composición de los gases.

- La temperatura de refrigeración adecuada del producto debe ser asegurada antes del almacenamiento y distribución. El monitoreo cuidadoso de la temperatura de almacenaje es crítico y debe formar parte de un programa de control de calidad.

- Las frutas deben ser puestas en almacenamiento tan pronto como sea posible después de la cosecha y en cualquier caso el mismo día.

- El cuarto debe ser sellado y enfriado una vez concluya la carga.

- Solamente un tipo de producto debe almacenarse en el mismo cuarto y preferiblemente de un mismo cultivo (Thompson, 1997).

- Presenta mejores resultados para frutas de tamaño pequeño y mediano (diámetro menor o igual a $10 \mathrm{~cm}$ ), pero no para frutas de gran tamaño.

- Es un proceso costoso, por lo que deben almacenarse solamente frutas en excelente condición.

\section{Generalidades de los productos hortofrutícolas}

Los productos hortofrutícolas son básicamente todos los productos de origen vegetal para consumo humano (frutas y hortalizas). Están formados por tejidos vivos que son metabólicamente activos los cuales cambian constantemente su composición, lo que hace que su conservación dependa de su filogismo y grado de madurez. Mientras el vegetal está unido a la planta recibe los beneficios de los procesos metabólicos de esta, donde la respiración de la planta intercambia eficazmente el $\mathrm{O}_{2}$ y el $\mathrm{CO}_{2}$ producidos, haciendo que las concentraciones de estos gases en los tejidos oscilen de acuerdo a la variedad y tamaño. Tal situación retarda la maduración del fruto, algo muy parecido al fenómeno de AC, por esta razón muchas frutas se pueden mantener por largo tiempo en la planta sin ser cosechadas. En la gráfica se muestran los intercambios que se presentan entre una fruta y el medio. 


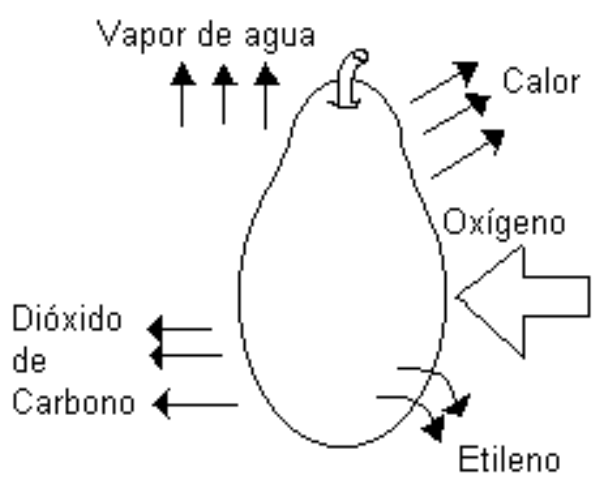

Figura 1. Intercambio entre la fruta y su entorno. Fuente: Seymour, G. Biochemistry of fruit ripening. Chapman \& Hall. London. 1998.

Al recolectar la fruta se interrumpe el flujo de savia y se genera un intercambio veloz de los gases con el aire exterior, por lo tanto el contenido interno de oxígeno sube aproximadamente al 20\%; el efecto del $\mathrm{CO}_{2}$ se limita y se acelera rápidamente el metabolismo. Esta actividad fisiológica que se presenta en los productos hortofrutícolas recolectados puede conducir en algunos casos a una disminución de calidad, mientras que en otros resulta necesario para lograr el grado deseado de maduración.

Una característica importante de los vegetales a tener en cuenta para su almacenamiento y conservación, es que, como seres vivos que son, respiran tomando oxígeno $\left(\mathrm{O}_{2}\right)$ y desprendiendo dióxido de carbono $\left(\mathrm{CO}_{2}\right)$ y calor, a la vez que también transpiran, es decir, pierden agua. La reacción química que se presenta en este proceso de respiración se puede observar en el siguiente balance:

$$
\mathrm{C}_{6} \mathrm{H}_{12} \mathrm{O}_{6}+6 \mathrm{O}_{2} \rightarrow 6 \mathrm{CO}_{2}+6 \mathrm{H}_{2} \mathrm{O}+\text { energía }
$$

$\mathrm{Al}$ recolectarse estos productos, se pierde la fuente de agua, algunos productos de la fotosíntesis y minerales, quedando así a disposición de sus propias reservas, consumiendo azúcares, produciendo una cantidad mayor de calor y expeliendo anhídrido carbónico que se disuelve en el aire; en este momento comienza una eliminación enzimática acelerada de la clorofila. Además se presenta una liberación de etileno el cual estimula adicionalmente el proceso de maduración.

La tasa respiratoria es un constituyente básico para determinar las velocidades de renovación de la atmósfera en el sitio de almacenamiento, la cual depende básicamente del tipo de producto, variedad, peso y el estado de madurez. Así mismo se determina la temperatura óptima de almacenamiento teniendo en cuenta que la velocidad de respiración se reduce de 2 a 3 veces al disminuir en $10^{\circ} \mathrm{C}$ la temperatura, también se reduce la cantidad de calor generado (Avilan, 1999). Otros factores que tienen que ver con la conservación y, que afectan la tasa de respiración son: Las presiones parciales de $\mathrm{O}_{2}$ y $\mathrm{CO}_{2}$, la humedad relativa y la luz. En el manejo del producto poscosecha, la tasa 
de respiración también se ve afectada por las lesiones del producto y por la carga microbiana.

\section{El Aguacate (Persea Americana)}

El aguacate se ha catalogado por muchos autores como el "rey de los frutos", pues es una fruta que posee gran cantidad de elementos nutritivos (glúcidos, vitaminas y minerales) más que ninguna otra, además de tener un sabor agradable.

Clase: Dicotiledóneas.

Orden: Ranales

Familia: Lauráceas.

Género: Persea

Especie: Americana (Miller)

Gratísima (Gaertner)

Origen: México.

Planta: árbol vigoroso, pudiendo alcanzar hasta $30 \mathrm{~m}$ de altura (Carvajal 1996).

El nombre de aguacate proviene de la palabra nativa "aocatl" o "ahuacatl". A partir de pruebas arqueológicas encontradas en Tehuacan (México), se ha determinado que es originario de México debido a que la antigüedad de dichas muestras se calcula en 12 000 años. A nivel mundial se comienza la explotación intensiva del aguacate con perspectivas comerciales y de mercado en el año 1932, siendo su principal productor México con más de 1.000.000 de toneladas anuales, de las cuales el 95\% es variedad Hass. Le sigue Estados Unidos (300.000 ton), Indonesia (225. 0000 ton), y Colombia (130.000 ton) (FAO 2001), iniciando actualmente la exportación de esta apetecida fruta.

Las perspectivas comerciales del aguacate han demostrado ser positivas. Actualmente es un cultivo en expansión, siendo las principales variedades de exportación el Hass y el nabal; por lo tanto su almacenamiento en condiciones óptimas cobra una importancia técnica y económica primordial. Es importante destacar que el aguacate posee valiosas propiedades alimenticias por su alto contenido de aceite (12 a 30\%) y proteínas (3 a $4 \%)$. Estas características le confieren grandes posibilidades en el aumento de su consumo en la dieta humana. Actualmente se esta desarrollando su industrialización en la producción de alimentos, extracción de aceites y productos farmacológicos.

\section{Características básicas del aguacate variedad Hass}

El Hass es la variedad de aguacate más comercializada en el mundo debido a sus ventajas productivas y a características como: pulpa cremosa de excelente sabor, ausencia de fibras, un $23 \%$ en contenido de aceites, cáscara roja y coriácea (más resistente al daño), color morado oscuro al madurar y semilla pequeña.

El fruto es de forma ovalada, la semilla es pequeña, pela fácilmente y de agradable sabor. Contrario a lo que se cree, el aguacate tiene un benéfico para la salud. Ayuda a contrarrestar los efectos del colesterol, ya que, disminuye los triglicéridos y aumenta las lipoproteínas de alta densidad. 
Uruapan, en México, es la capital mundial del aguacate. Allí se producen anualmente 700.000 toneladas de la variedad Hass. Cien gramos de pulpa Hass contienen proteínas, ácidos saturados, retinol (A), tiamina, riboflavina, vitaminas C, E, B6, ácido pantotéico, calcio, hierro, magnesio, sodio, potasio y zinc. En la tabla se muestra el análisis de 100 gramos de pulpa de aguacate Hass.

\section{Consideraciones técnicas de almacenamiento de aguacate (Persea americana) variedad Hass en atmósferas controladas}

La maduración del aguacate está asociada con el incremento en la velocidad de respiración, la cual es favorecida por la producción interna o la aplicación externa del etileno. Para disminuir la respiración de la fruta esta debe someterse a un enfriamiento en las 6 horas siguientes a su recolección (Bennett 1987), en caso de no ser posible se recomienda mantener su temperatura por debajo de los $25^{\circ} \mathrm{C}$.

El aguacate es un fruto sensible a los daños por frío (DF) cuando es sometido a bajas temperaturas por tiempos prolongados. Los principales síntomas del DF se manifiestan como zonas cafés en la cáscara y una decoloración que va desde el café tenue hasta el café oscuro en el mesocarpio, atribuido a la enzima polifenol oxidasa (PPO). Se recomienda por lo tanto que la refrigeración a $7^{\circ} \mathrm{C}$ no exceda más de 30 a 40 días ya que los niveles de PPO aumentan a medida que el almacenamiento se prolonga. El oscurecimiento interno es un factor limitante para prolongar la vida de anaquel del aguacate Hass por más de dos semanas a $7^{\circ} \mathrm{C}$. Las variedades Fuertes, Hass y Nabal se caracterizan por tener una tolerancia a las bajas temperaturas de almacenamiento. A $4^{\circ} \mathrm{C}$ estas variedades pueden almacenarse por 2 ó 4 semanas (Kadan, 1995).

En un estudio efectuado por Hopkirt (1998) se observó que el aguacate variedad Hass madurado a $20^{\circ} \mathrm{C}$, presentaba menos pudriciones cuando previamente se almacenaba en cámaras de atmósferas controladas a temperaturas comprendidas entre 4 y $6^{\circ} \mathrm{C}$, que cuando permanecía a temperaturas mayores o menores a las descritas, concluyendo que la mejor calidad en el fruto fue lograda almacenándolo en cámaras de atmósfera modificada a $6^{\circ} \mathrm{C}$.

Las zonas productoras del aguacate en el mundo se encuentran lejanas de las zonas de comercio, por lo que es importante retardar la maduración entre la cosecha y la llegada del producto al punto de consumo. Es necesario desarrollar una adecuada tecnología de almacenamiento para retardar la maduración y mantener una óptima calidad. Entre las más destacadas está la del almacenamiento en cámaras de atmósfera controladas.

Altas concentraciones de $\mathrm{CO}_{2}$ y Bajas de $\mathrm{O}_{2}$ en la atmósfera retardan la maduración (ablandamiento y cambio de color en el exocarpio) y disminuyen los síntomas de DF durante el almacenamiento. Las concentraciones adecuadas para el aguacate varían entre $5-8 \%$ de $\mathrm{CO}_{2}$ y de $3-5 \%$ de $\mathrm{O}_{2}$. Posterior al almacenamiento en atmósferas controladas el fruto tiende a seguir su maduración normal.

En la variedad Hass las cámaras de atmósferas controladas disminuyen el deterioro del fruto y el daño por frío, además de prolongar la vida poscosecha al reducir la perdida de peso y el ablandamiento de la pulpa. El nivel recomendado de $\mathrm{O}_{2}$ para esta variedad es de $4 \%$ y de $5 \%$ para el $\mathrm{CO}_{2}$. La de $\mathrm{N}_{2}$ es del $90 \%$ a una temperatura de $7^{\circ} \mathrm{C}$ para un 
almacenamiento de cinco semanas (Hopkirt, 1998). Es importante anotar que un nivel mayor del $5 \%$ de $\mathrm{CO}_{2}$ puede incrementar el oscurecimiento de la pulpa y la presencia de concentraciones bajas de etileno (1ppm) en la atmósfera puede anular los efectos de la atmósfera controlada.

La humedad relativa presente en la cámara también debe ser tenida en cuenta, la cual debe ser del 92\%; ya que es importante saber la proporción del vapor de $\mathrm{H}_{2} \mathrm{O}$ presente en la atmósfera pues la presión ejercida por el vapor en el aire y la presión ejercida por el $\mathrm{H}_{2} \mathrm{O}$ presente en los tejidos de la fruta generan una diferencia conocida como el déficit de presión de vapor (DPV), el cual determina la intensidad de transpiración del producto, El DPV dentro de una cámara de atmósfera controlada debe ser mínimo para que no se presente la deshidratación de la fruta.

\section{Bibliografía}

Artes, F. Conservación de productos hortofrutícolas en atmósferas controladas y modificadas. V Curso superior Ingeniería y aplicación del frío en la conservación de vegetales. 1999.

[D] Avilan, L. Sistema de plantación de altas densidades en aguacate. Agronomía tropical. $\mathrm{N}^{\mathrm{o}}$ 27. p $479-484$. 1999.

[D] Bartsch, James y BLANPIED, David. Refrigeration and controlled atmosphere storage for horticultural crops. ITACA, N.Y.: Northeast Regional Agricultural Engineering Service. 45 p. 1990.

[D] Bennett, A. Regulation of climacteric respiration in ripening avocado fruit. Plan physiol. 1987.

[D] Brecht, P E. Use of controlled atmospheres to retard deterioration of produce. Food technology. No 34. Páginas 45 - 50. 1980.

[D] Carvajal M. Juan Guillermo. Manual práctico para el cultivo del aguacate. SENA (Rionegro, Antioquia). 1996.

[D] Corrales G, Joel; Hernández M., Juan y Gómez V., Raúl. Uso comercial de las atmósferas controladas en México. M México: Universidad autónoma de Chapingo. 1990.

[D] Comunidad Europea. Legislación alimentaria. En: http://europa.eu.int/. Octubre 2002.

D] Duckworth, R. B Frutas y verduras. Zaragoza España: Editorial Acribia, 1968.

[D] FAO. Stadistical Database. www.fao.org . 2002.

[Da] Garret, M.E. Applications of controlled atmosphere containers. Guilford, England: July 1995.

[C] Gil, M I; Artes, F; Tomas Barberan; F. A. Minimal processing and modified atmosphere packaging effects on pigmentation of pomegranate seeds. EN: Journal of Food Science, Vol 61. No 1. 161-164. 1996.

[D] Gobernación de Antioquia. Frutas de Colombia para el mundo manual para el exportador. Secretaría de agricultura. Programa de las naciones unidas para el desarrollo. Medellín, 1991.

[D] Hochhaus, K. H. y Wild, Y. Transporte de productos refrigerados en atmósfera controlada. En: International fruit world. No 1, No 2, No 3. Pág 312-321, 1993.

[D] Hopkirt, G. Influence of post harvest temperatures and the rate of fruit ripening on internal post harvest rots of New Zeeland Hass avocado fruit. 1998. 
[D] Jameson, J. Tecnología de conservación en la atmósfera controlada. Últimos desarrollos y perspectivas del futuro. En: Frío Calor y Aire acondicionado No 278. 1987.

[Dd Kadan, S. Avocado. Marcel Dekker (Ed). 1995.

[D] Lougheed, E. C. Small scale simulated commercial C.A. storage rooms. North Carolina State University. Report No 126.1985.

[Da] Meneses A. Miguel. Planeación y operación de cuartos fríos para frutas y hortalizas. SENA - Reino Unido. 2000.

[D] Parry, R T. Envasado de los alimentos en atmósferas modificadas. A. Madrid Vicente, Ediciones. 1993.

[C] Proexpo. Frutas y hortalizas frescas. Bogotá: Dpto de información y publicaciones. 1989.

[D] Refrigeration Institute. Refrigeración y aire acondicionado. Madrid España: Editorial Dossat S.A: 1981.

[D] Reunión técnica de la red latinoamericana de agroindustria de frutas tropicales "producción, manejo y exportación de frutas tropicales", Federación nacional de cafeteros de Colombia, oficina regional de la FAO para América Latina y el Caribe. 1987.

[D] Roy, S; Anantheswaran; R.C.; Beelman, R. B. Modified atmosphere and midiefied humidity packaging of fresh mushrooms. En: Journal of Food Science, Vol 61 No 2, p. 391-397. 1996.

[D] Seymour, G. Biochemestry of fruit ripening. Chapman \& Hall. London. 1993.

[D] Thompson, A. K. Postharvest technology of fruits and vegetables. Oxford: Blackwell. 327 p. 1996.

[Dd Turay, Samuel. Transporte refrigerado de productos perecederos. En: Frío Calor y Aire acondicionado No 314. 2000.

[Da Will, R. H; Lee T. H. Fisiología y manipulación de frutas y hortalizas post recolección. Zaragoza, España: Editorial Acribia, 1982.

[Dd Zagory, D.; Kader, A. A. Modified atmosphere packaging of fresh produce. EN: Food Technology, September, p. 70-77. 1988. 